\title{
REPRESENTATION THEORY IN COMPLEX RANK, I
}

\author{
PAVEL ETINGOF
}

\section{Dedicated to E. B. Dynkin on his 90th birthday}

\section{INTRODUCTION}

The subject of representation theory in complex rank goes back to the papers [DM, De1. Namely, these papers introduce Karoubian tensor categories $\operatorname{Rep}\left(G L_{t}\right)([\mathrm{DM}, \mathrm{De} 1]), \operatorname{Rep}\left(O_{t}\right), \operatorname{Rep}\left(S p_{2 t}\right), t \in \mathrm{C}$ ([De1]), which are interpolations of the tensor categories of algebraic representations of classical complex algebraic groups $G L_{n}, O_{n}, S p_{2 n}$ to non-integral rank 1 . This means that when $t=n$ is a nonnegative integer, these categories project onto the corresponding classical representation categories $\boldsymbol{\operatorname { R e p }}\left(G L_{n}\right), \boldsymbol{\operatorname { R e p }}\left(O_{n}\right), \boldsymbol{\operatorname { R e p }}\left(S p_{2 n}\right)$, i.e., they have tensor ideals, the quotients by which are the classical representation categories. Later, in [De2], P. Deligne introduced Karoubian tensor categories $\operatorname{Rep}\left(S_{t}\right), t \in \mathrm{C}$, which are similar interpolations for the representation category of the symmetric group $\operatorname{Rep}\left(S_{n}\right)$ (and project onto it for $t=n$ ).

In [Kn1, Kn2], F. Knop proposed a broad generalization of Deligne's construction. In particular, he generalized his construction for $S_{n}$ to the case of wreath product groups $S_{n} \ltimes \Gamma^{n}$, where $\Gamma$ is any finite group, constructing Karoubian tensor categories $\operatorname{Rep}\left(S_{t} \ltimes \Gamma^{t}\right)$ for complex $t$, projecting for $t=n$ onto $\operatorname{Rep}\left(S_{n} \ltimes \Gamma^{n}\right)$.

Since these categories are semisimple for non-integer $t$, one may think of these results as "compact" representation theory in complex rank. The goal of this paper is to start developing the "noncompact" counterpart of this theory. Namely, in this paper we will introduce a method that allows one to define interpolations to complex rank of various categories of representations of classical type, in particular the following ones:

1) wreath products;

2) degenerate affine Hecke algebras;

3) rational and trigonometric Cherednik algebras, symplectic reflection algebras;

4) real groups (i.e., symmetric pairs);

\footnotetext{
${ }^{1}$ In fact, $\operatorname{Rep}\left(O_{t}\right)=\operatorname{Rep}\left(S p_{-t}\right)$ with a modified symmetric structure.
} 
5) Lie superalgebras;

6) affine Lie algebras;

7) Yangians;

8) (Parabolic) category $\mathrm{O}$ for reductive Lie algebras.

Namely, we will define representations of a "noncompact algebra" of complex rank as representations of its "maximal compact subalgebra" (i.e. an (ind)-object of the corresponding tensor category) together with some additional structure (morphisms satisfying some relations). These morphisms and relations are obtained by writing down a "categorically friendly" definition of the corresponding classical structure, and then interpolating this definition to complex values of the rank parameter(s). We will discuss the explicit form of such morphisms and relations in the above special cases $1-3$, based on $\operatorname{Rep}\left(S_{t}\right)$. Cases 4-8 based on $\operatorname{Rep}\left(G L_{t}\right), \operatorname{Rep}\left(O_{t}\right), \operatorname{Rep}\left(S p_{2 t}\right)$ and many other similar situations will be considered in future papers. 2

This approach leads to a multitude of new interesting representation categories, which, in a sense, capture the phenomenon of "stabilization with respect to rank" in representation theory of classical groups and algebras. In subsequent works, we plan to study the structure of these categories in detail. We expect to discover interesting degeneration phenomena not only at integer $t$, but also at rational (non-integer) values of $t$. Specifically, if the "classical" theory already has a continuous parameter $k$ (or several such parameters), then we expect interesting degeneration phenomena when both $k$ and $t$ are rational but not necessarily integer. Such phenomena cannot be understood by interpolation arguments, and their study will likely require new ideas. For instance, for rational Cherednik algebras, such degeneration phenomena were discovered in [EA1]. Also, one might hope that these new categories will be interesting for the theory of categorification.

For simplicity, we consider only the case $t \notin \mathrm{Z}_{+}$, when the Deligne categories $\operatorname{Rep}\left(S_{t}\right)$ are semisimple, although many of the results admit generalizations to the case $t \in Z_{+}$.

The organization of the paper is as follows.

In Section 2 we recall basic facts about the Deligne category $\operatorname{Rep}\left(S_{t}\right)$. In particular, we define the group algebra C $\left[S_{t}\right]$ of $S_{t}$ and discuss its center, and its action on simple objects of $\operatorname{Rep}\left(S_{t}\right)$.

In Section 3, we discuss the Schur-Weyl dualty for $\operatorname{Rep}\left(S_{t}\right)$, using the notion of a complex tensor power of a vector space with a distinguished nonzero vector.

\footnotetext{
${ }^{2}$ The ideas explored in this paper were outlined in the talk $\mathrm{E}$ in March 2009.
} 
In Section 4, we apply the Schur-Weyl duality from Section 3 to define and study wreath products of complex rank.

Finally, in Section 5 we discuss interpolations of degenerate affine Hecke algebras and of wreath product symplectic reflection algebras, in particular rational Cherednik algebras.

Remark 1.1. The world of representation categories of complex rank is vast and almost unexplored, and there are a multitude of projects that immediately spring to mind, to interpolate various settings in classical representation theory. Many of them seem interesting, and it is not yet clear which ones are most worthy of exploration.

It seems that a good strategy of choosing problems in this field at initial stages would be to keep closer to applications to "classical" representation theory. One of the pathways for such applications is to consider the algebra $A=\operatorname{End}(X)$, where $X$ is an (ind-)object of some complex rank category $\mathcal{C}$. This is an ordinary algebra that belongs to the world of "classical" representation theory, but its construction as $\operatorname{End}(X)$ is sometimes more insightful than "classical" constructions, if they exist at all. In particular, through such a construction, $A$ comes equipped with a large family of modules, namely $\operatorname{Hom}(X, Y)$, where $Y$ is another object of $\mathcal{C}$.

Another principle that might be useful is to focus on extracting concrete numerical information (such as characters, multiplicities, etc.), as this has been a fundamental principle of representation theory since its creation. To this end, it may be useful to relate complex rank categories to "classical" representation categories through various functorial constructions, such as Schur-Weyl duality and its generalizations.

Acknowledgments. The author is grateful to I. Entova-Aizenbud and V. Ostrik for many useful discussions. The work of the author was partially supported by the NSF grants DMS-0504847 and DMS1000113.

\section{Deligne Categories $\operatorname{Rep}\left(S_{t}\right)$}

2.1. Categorical preliminaries. All categories we consider will be additive and linear over $\mathrm{C}$.

Recall that a Karoubian category is an additive category closed under taking direct summands.

By a tensor category, unless otherwise specified, we will mean a Karoubian rigid monoidal category with additive tensor product. 
For a category $\mathcal{C}$ denote by $\operatorname{Ind} \mathcal{C}$ the ind-completion of $\mathcal{C}$. If $\mathcal{C}$ is semisimple, objects of $\operatorname{Ind}(\mathcal{C})$ are possibly infinite direct sums of indecomposable objects of $\mathcal{C}$. By an ind-object in $\mathcal{C}$ we will mean an object of $\operatorname{Ind} \mathcal{C}$.

If $\mathcal{C}, \mathcal{D}$ are semisimple categories, by $\mathcal{C} \otimes \mathcal{D}$ we denote their external tensor product, - a semisimple category whose simple objects have the form $X \otimes Y$, where $X$ runs over simple objects of $\mathcal{C}$ and $Y$ over simple objects of $\mathcal{D}$. It is clear that if $\mathcal{C}, \mathcal{D}$ are tensor categories then the category $\mathcal{C} \otimes \mathcal{D}$ is also a tensor category.

2.2. Definition of the Deligne category $\operatorname{Rep}\left(S_{t}\right)$ and its basic properties. Let us recall basic facts about the Deligne category $\operatorname{Rep}\left(S_{t}\right)$ ([De1]). 3 This is a Karoubian rigid tensor category over C defined for any complex number $t$. Indecomposable objects $X_{\lambda}$ of the category $\operatorname{Rep}\left(S_{t}\right)$ are labeled by all Young diagrams $\lambda$.

If $t$ is not a nonnegative integer, then $\operatorname{Rep}\left(S_{t}\right)$ is semisimple abelian, and its simple objects $X_{\lambda}$ are labeled by all Young diagrams (or partitions) $\lambda$. In particular, the empty diagram corresponds to the neutral object $\mathbf{1}$, the one-box diagram to the "reflection representation" $\mathfrak{h}_{0}$, the column of length $k$ to $\wedge{ }^{k} \mathfrak{h}_{0}$, etc. The object $X_{\lambda}$ interpolates the representations of $S_{n}$ corresponding to the partition

$$
\widetilde{\lambda}(n):=\left(n-|\lambda|, \lambda_{1}, \ldots, \lambda_{m}, \ldots\right)
$$

of $n$, for large $n$.

On the other hand, if $t$ is a nonnegative integer $n$, then $\operatorname{Rep}\left(S_{t}\right)$ projects onto the category of representations of the symmetric group $\boldsymbol{\operatorname { R e p }}\left(S_{n}\right)$, whose simple objects are the irreducible representations $\pi_{\mu}$ of $S_{n}$ attached to Young diagrams (or partitions) $\mu$ with $|\mu|=n$. Namely, $\operatorname{Rep}\left(S_{t}\right)$ contains a tensor ideal $\mathcal{I}_{n}$, such that $\operatorname{Rep}\left(S_{t}\right) / \mathcal{I}_{n}=\operatorname{Rep}\left(S_{n}\right)$. The partitions $\lambda$ for which $X_{\lambda}$ have a nonzero image in the quotient are those for which $\lambda_{1}+|\lambda| \leq n$, and for such $\lambda$ the object $X_{\lambda}$ maps to $\pi_{\widetilde{\lambda}(n)} \in \operatorname{Rep}\left(S_{n}\right)$.

Deligne also defined the abelian category $\operatorname{Rep}^{a b}\left(S_{t}\right)$, which is "the abelian envelope" of $\operatorname{Rep}\left(S_{t}\right)$ (it differs from $\operatorname{Rep}\left(S_{t}\right)$ only for nonnegative integer $t$ ).

The categorical dimensions of indecomposable objects in the category $\operatorname{Rep}\left(S_{t}\right)$ for $t \notin \mathrm{Z}_{+}$are given by the following formula. For each partition $\lambda$ with $|\lambda|=N$, let $\lambda^{*}$ be the conjugate partition, and define the set $B_{\lambda}$ to be the set of all nonnegative integers not contained in the

\footnotetext{
${ }^{3} \mathrm{~A}$ good source of materials on Deligne categories is the webpage of the MIT seminar on Deligne categories, http://math.mit.edu/ ${ }^{\sim}$ innaento/DeligneCatSeminar/
} 
sequence $N-1+k-\lambda_{k}^{*}, k \geq 1$ (since this sequence is increasing, it is clear that $\left.\left|B_{\lambda}\right|=N\right)$. Then we have

Proposition 2.1. (see [De2], CO]) If $t \notin \mathrm{Z}_{+}$, then

$$
\operatorname{dim} X_{\lambda}=\operatorname{dim} \pi_{\lambda} \frac{\prod_{k \in B_{\lambda}}(t-k)}{N !}=\frac{\prod_{k \in B_{\lambda}}(t-k)}{\prod_{(i, j) \in \lambda} h_{\lambda}(i, j)},
$$

where $h_{\lambda}(i, j)$ are the hooklengths in $\lambda$.

This formula is obtained by interpolating the hooklength formula for the dimensions of $\pi_{\widetilde{\lambda}(n)}$.

Example 2.2. 1. Let $\lambda=\left(1^{k}\right)$ ( $k$ times). Then $\operatorname{dim} X_{\lambda}=\frac{(t-1) \ldots(t-k)}{k !}=$ $\left(\begin{array}{c}t-1 \\ k\end{array}\right)$.

2. Let $\lambda=(k)$. Then $\operatorname{dim} X_{\lambda}=\frac{(t-2 k+1) \prod_{j=0}^{k-2}(t-j)}{k !}=\left(\begin{array}{l}t \\ k\end{array}\right)-\left(\begin{array}{c}t \\ k-1\end{array}\right)$.

Remark 2.3. Formula (2.1) is not always true for nonnegative integer $t$. For instance, as we see from Example 2.2(2), the polynomial giving $\operatorname{dim} X_{(k)}$ for generic $t$ is negative for $t=k-1, \ldots, 2 k-2$. This means that $\operatorname{dim} X_{(k)}$ cannot be given by this formula for these values of $k$ (as then it will have nothing to be mapped to in the category of representations of the symmetric group, as the functor from $\operatorname{Rep}\left(S_{t}\right)$ to this category is a symmetric tensor functor and hence preserves dimensions). In fact, one can show (see [CO]) that $X_{\lambda}$ for positive integers $t$ is not always a specialization of a simple object for generic $t$; its lift to generic $t$ may be reducible.

It is also useful to recall the rule of multiplication by $\mathfrak{h}_{0}$.

Proposition 2.4. (see [De2, [CO]) One has

$$
\mathfrak{h}_{0} \otimes X_{\lambda}=\oplus_{\mu \in P_{\lambda}^{+} \cup P_{\lambda}^{-} \cup P_{\lambda}^{0}} X_{\mu}+\operatorname{cc}(\lambda) X_{\lambda},
$$

where $P_{\lambda}^{+}, P_{\lambda}^{-}, P_{\lambda}^{0}$ are the sets of Young diagrams obtained from $\lambda$ by adding, deleting, and moving a corner cell, respectively, and $\operatorname{cc}(\lambda)$ is the number of corner cells of $\lambda$.

This formula is obtained by interpolating the Pieri rule.

2.3. The universal property, induction, and restriction. For $t \notin$ $\mathrm{Z}_{+}$, the category $\operatorname{Rep}\left(S_{t}\right)$ is known (see [De1], Section 8) to be the universal symmetric tensor category with a commutative Frobenius algebra $\mathfrak{h}$ of dimension $t$. This means that for any symmetric tensor category $\mathcal{C}$, tensor functors $\operatorname{Rep}\left(S_{t}\right) \rightarrow \mathcal{C}$ correspond of commutative Frobenius algebras in $\mathcal{C}$ of dimension $t$. 
Now let $t_{1}, \ldots, t_{m} \in \mathrm{C}$ be such that $\sum_{i=1}^{m} t_{i}=t$. Consider the category $\bigotimes_{i=1}^{m} \operatorname{Rep}\left(S_{t_{i}}\right)$. In this category we have the commutative Frobenius algebra $\mathfrak{h}_{1} \oplus \ldots \oplus \mathfrak{h}_{m}$, where

$$
\mathfrak{h}_{i}:=\mathbf{1}^{\bigotimes i-1} \otimes \mathfrak{h}_{\operatorname{Rep}\left(S_{t_{i}}\right)} \otimes \mathbf{1}^{\bigotimes m-i} .
$$

The dimension of this algebra is $t$. So by virtue of the universal property, we have a symmetric tensor functor

$$
\operatorname{Res}_{t_{1}, \ldots, t_{n}}: \operatorname{Rep}\left(S_{t}\right) \rightarrow \bigotimes_{i=1}^{m} \operatorname{Rep}\left(S_{t_{i}}\right),
$$

such that $\operatorname{Res}_{t_{1}, \ldots, t_{n}}(\mathfrak{h})=\mathfrak{h}_{1} \oplus \ldots \oplus \mathfrak{h}_{m}$. The left (respectively, right) adjoint of this functor is called the induction (respectively, coinduction) functor, and denoted by (Co) $\operatorname{Ind}_{t_{1}, \ldots, t_{m}}$ (it lands in the ind-completion of $\left.\operatorname{Rep}\left(S_{t}\right)\right)$. In the special case $m=2$ and $t_{2} \in \mathrm{Z}_{+}\left(\right.$with $\operatorname{Rep}\left(S_{t_{2}}\right)$ instead of $\operatorname{Rep}\left(S_{t_{2}}\right)$ ), these functors are considered in [De1].

2.4. The Jucys-Murphy central element. Recall that the JucysMurphy central element is the central element $\Omega=\sum_{1 \leq i<j \leq n} s_{i j}$ in C $\left[S_{n}\right]$, where $s_{i j}$ is the transposition of $i$ and $j$. It is well known that $\Omega$ acts on an irreducible representation $\pi_{\mu}$ by the scalar ct $(\mu)$ (the content of $\mu$ ), which is the sum of $i-j$ over all cells $(i, j)$ of the Young diagram $\mu$.

The interpolation of the Jucys-Murphy central element to complex values of $t$ (as an endomorphism of the identity functor of the category $\operatorname{Rep}\left(S_{t}\right)$ ) is constructed in the paper [CO] (as a special case of one-cycle central elements, see below). When $t \notin \mathrm{Z}_{+}, \mathrm{t}$ is easy to describe this endomorphism explicitly. Indeed, it is easy to see that

$$
\operatorname{ct}(\tilde{\lambda}(n))=\operatorname{ct}(\lambda)-|\lambda|+\frac{(n-|\lambda|)(n-|\lambda|-1)}{2} .
$$

So we define the endomorphism $\Omega$ by the formula

$$
\left.\Omega\right|_{X_{\lambda}}:=\operatorname{ct}(\lambda)-|\lambda|+\frac{(t-|\lambda|)(t-|\lambda|-1)}{2} .
$$

2.5. Higher central elements. In a similar way one can interpolate other central elements of the group algebra $\mathrm{C}\left[S_{n}\right]$, corresponding to various cycle types. Namely, let $\mathbf{m}=\left(m_{1}, m_{2}, \ldots\right)$ be a sequence of nonnegative integers with $\sum m_{i}(i+1) \leq n$. Let $m=\sum m_{i}(i+1)$. Then in the group algebra $\mathrm{C}\left[S_{n}\right]$ we have the central element $\Omega_{\mathbf{m}}$, which is the sum of all permutations with $m_{i}$ cycles of length $i+1$ for each $i \geq 1$. The eigenvalue of $\Omega_{\mathbf{m}}$ on $\pi_{\lambda}$ equals

$$
\left.\Omega_{\mathbf{m}}\right|_{\pi_{\lambda}}=\frac{\left.\left|C_{\mathbf{m}}\right| \cdot \operatorname{tr}\right|_{\pi_{\lambda}}(g)}{\operatorname{dim} \pi_{\lambda}}
$$


where $C_{\mathbf{m}}$ is the conjugacy class of permutations with $m_{i}$ cycles of length $i+1$ for each $i \geq 1$, and $g \in C_{\mathbf{m}}$. We have

$$
\left|C_{\mathbf{m}}\right|=\frac{n(n-1) \ldots(n-m+1)}{\prod_{i} m_{i} !(i+1)^{m_{i}}} .
$$

This implies that the interpolation of $\Omega_{\mathbf{m}}$ to $\operatorname{Rep}\left(S_{t}\right)$ is given by the formula

$$
\left.\Omega_{\mathbf{m}}\right|_{X_{\lambda}}=\frac{\prod_{(i, j) \in \lambda} h_{\lambda}(i, j) \cdot \prod_{j=0}^{m-1}(t-j)}{\prod_{i} m_{i} !(i+1)^{m_{i}} \cdot \prod_{k \in B_{\lambda}}(t-k)} c_{\lambda, \mathbf{m}}(t)
$$

where $c_{\lambda, \mathbf{m}}(t)$ is the coefficient of $x^{\lambda}:=\prod_{i} x_{i}^{\lambda_{i}}$ in the series

$$
F_{t, \mathbf{m}}(x):=\left(1+p_{1}(x)\right)^{t-m} \prod_{i \geq 1}\left(1+p_{i+1}(x)\right)^{m_{i}} \prod_{i \geq 1}\left(1-x_{i}\right) \prod_{i>j}\left(1-\frac{x_{i}}{x_{j}}\right),
$$

where $p_{i}(x)=\sum_{r} x_{r}^{i}$ (it is clear that $c_{\lambda, \mathbf{m}}(t)$ is a polynomial, by setting $x_{i}=u_{1} \ldots u_{i}$ and writing the expansion in terms of the $\left.u_{i}\right)$. This interpolation is obtained from the Frobenius character formula and the hooklength formula for $S_{n}$-representations, and for the case of one cycle it is given in [CO]. In particular, $\Omega_{1,0,0 \ldots}=\Omega$ (the Jucys-Murphy element) 4

Note that $\left.\Omega_{\mathbf{m}}\right|_{X_{\lambda}}$, as well as $\operatorname{dim} X_{\lambda}$, is an integer-valued polynomial of $t$ (i.e., an integer linear combination of binomial coefficients). Indeed, this function takes integer values at large positive integers $t$ (by representation theory of symmetric groups), and such a rational function is well known to be an integer-valued polynomial.

2.6. The group algebra $\mathrm{C}\left[S_{t}\right]$ of $S_{t}$. The representation category of the symmetric group $S_{n}$ may be defined as the tensor category of representations of the Hopf algebra $\mathrm{C}\left[S_{n}\right]$. In the setting of Deligne categories, such an algebra can also be defined, as a Hopf algebra in $\operatorname{Rep}\left(S_{t}\right)$ (which interpolates the group algebra of $S_{n}$ with the conjugation action of $S_{n}$ ). The only caveat is that since $\left|S_{n}\right|=n$ ! is not a polynomial in $n$, this algebra will be infinite dimensional (i.e., an ind-object of $\operatorname{Rep}\left(S_{t}\right)$ ).

Namely, let us fix a cycle type $\mathbf{m}$ as above. Then we have the conjugacy class $C_{\mathbf{m}}$ in $S_{n}$, and the span of this conjugacy class in

\footnotetext{
${ }^{4}$ Note that the element $\Omega_{\mathbf{m}}$ is a polynomial of the elements $Z_{1}, Z_{2}, \ldots$, where $Z_{i}:=\Omega_{\mathbf{e}_{i}}$ are the one-cycle central elements discussed in $[\mathrm{CO}]\left(\left(\mathbf{e}_{i}\right)_{j}=\delta_{i j}\right)$. For this reason, the one-cycle elements suffice for the study of blocks of $\operatorname{Rep}\left(S_{t}\right)$, done in $\mathrm{CO}$; more general central elements don't carry additional information.
} 
C $\left[S_{n}\right]$ is a representation of $S_{n}$ (with the conjugation action). This representation is the induced representation

$$
\operatorname{Ind}_{S_{n-m} \times \prod S_{m_{i}} \ltimes(\mathrm{z} /(i+1) \mathrm{z})^{m_{i}}}^{S_{n} \mathrm{C}} .
$$

(where $S_{m_{i}} \ltimes\left(\mathrm{z} /(i+1)\right.$ is viewed as a subgroup of $\left.S_{n}\right)$ This induced representation has a natural analog in $\operatorname{Rep}\left(S_{t}\right)$, namely the invariants $E_{\mathbf{m}}$ of the group $\prod_{i} S_{m_{i}} \ltimes(\mathrm{z} /(i+1) \mathrm{z})^{m_{i}}$ in the object $\Delta_{m, t}=\operatorname{Ind}_{S_{t-m}}^{S_{t}}(\mathbf{1})$ (where $\operatorname{Ind}_{S_{t-m}}^{S_{t}}$ is the induction functor $\operatorname{Rep}\left(S_{t-m}\right) \rightarrow \operatorname{Rep}\left(S_{t}\right)$ ). We also have a natural multiplication map

$$
\text { mult }_{\mathbf{m}, \mathbf{m}^{\prime}, \mathbf{m}^{\prime \prime}}: E_{\mathbf{m}} \otimes E_{\mathbf{m}^{\prime}} \rightarrow E_{\mathbf{m}^{\prime \prime}},
$$

which interpolates the multiplication in $\mathrm{C}\left[S_{n}\right]$ (i.e., in the classical setting for $s \in C_{\mathbf{m}}, s^{\prime} \in C_{\mathbf{m}^{\prime}}$ one has $\operatorname{mult}_{\mathbf{m}, \mathbf{m}^{\prime}, \mathbf{m}^{\prime \prime}}\left(s, s^{\prime}\right)=s s^{\prime}$ if $s s^{\prime} \in C_{\mathbf{m}^{\prime \prime}}$, and zero otherwise, and we need to interpret this in terms of the diagrams of the partition algebra). Moreover, it is clear that for fixed $\mathbf{m}$ and $\mathbf{m}^{\prime}$ this map is zero for almost all $\mathbf{m}^{\prime \prime}$. This shows that $\mathrm{C}\left[S_{t}\right]:=\oplus_{\mathbf{m}} E_{\mathbf{m}}$ is an associative algebra in $\operatorname{IndRep}\left(S_{t}\right)$. Note that this algebra is Z/2Z-graded by "parity of the permutation", $\operatorname{deg}\left(E_{\mathbf{m}}\right)=$ $\sum_{i} i m_{i} \bmod 2$.

Moreover, $\mathrm{C}\left[S_{t}\right]$ is a cocommutative Hopf algebra, in which the coproduct $\Delta: E_{\mathbf{m}} \rightarrow E_{\mathbf{m}} \otimes E_{\mathbf{m}}$ and counit $\varepsilon: E_{\mathbf{m}} \rightarrow \mathbf{1}$ come from the natural commutative algebra structure on $E_{\mathbf{m}}^{*}$ (note that $E_{\mathbf{m}}^{*}$ interpolates the space of functions on $C_{\mathbf{m}}$ ). It is also easy to construct the antipode $S: E_{\mathbf{m}} \rightarrow E_{\mathbf{m}}$, interpolating the inversion map on $S_{n}$. Namely, this is the antiautomorphism which is the identity on $E_{1,0, \ldots}$. Thus, the category C $\left[S_{t}\right]$-mod of C $\left[S_{t}\right]$-modules in $\operatorname{IndRep}\left(S_{t}\right)$ is a symmetric tensor category.

Note that we have a canonical tensor functor $\operatorname{Rep}\left(S_{t}\right) \rightarrow \mathrm{C}\left[S_{t}\right]-\bmod$; in particular, any object of $\operatorname{Rep}\left(S_{t}\right)$ carries a canonical action of c $\left[S_{t}\right]$. Indeed, to prescribe such a functor, it suffices to specify a Frobenius algebra in $\mathrm{C}\left[S_{t}\right]$-mod of dimension $t$. For this, it suffices to define a Hopf action of $\mathrm{C}\left[S_{t}\right]$ on the Frobenius algebra $\mathfrak{h}$, which is done in a straightforward way by interpolating from integer $n$ the standard action of $\mathrm{C}\left[S_{n}\right]$ on $\operatorname{Fun}(\{1, \ldots, n\})$.

Remark 2.5. Let $B$ be an algebra in $\operatorname{IndRep}\left(S_{t}\right)$. Then, since $B$ is an object of $\operatorname{IndRep}\left(S_{t}\right)$, there is a standard action of $\mathrm{C}\left[S_{t}\right]$ on $B$. It is easy to check that this is a Hopf algebra action of the Hopf algebra $H:=\mathrm{C}\left[S_{t}\right]$ on the algebra $B$ inside $\operatorname{IndRep}\left(S_{t}\right), \rho: H \otimes B \rightarrow B$. Thus, we can form the semidirect product $\mathrm{C}\left[S_{t}\right] \ltimes B$, as we do in the theory of Hopf algebra actions on rings. Namely, as an object, this semidirect product is $B \otimes H$, with multiplication map $m: B \otimes H \otimes B \otimes H \rightarrow B \otimes H$ 
is defined by the formula

$$
m=\left(m_{H} \otimes m_{B}\right) \circ(1 \otimes \rho \otimes 1 \otimes 1) \circ \sigma_{34} \circ\left(1 \otimes \Delta_{H} \otimes 1 \otimes 1\right),
$$

where $m_{B}, m_{H}$ are the products in $B, H, \Delta_{H}$ is the coproduct in $H$, and $\sigma_{34}$ is the permutation of the $3 \mathrm{~d}$ and 4 th components. Now, the category of $B$-modules in $\operatorname{IndRep}\left(S_{t}\right)$ is tautologically equivalent to the category of $\mathrm{C}\left[S_{t}\right] \ltimes B$-modules, in which $\mathrm{C}\left[S_{t}\right]$ acts via the standard action. 5 Moreover, if $B$ is a Hopf algebra then so is $\mathrm{C}\left[S_{t}\right] \ltimes B$, and the above equivalence of categories is a tensor equivalence.

Remark 2.6. It is easy to see that for each $\mathbf{m}, E_{\mathbf{m}}$ has a unique invariant up to scaling, which we denote by $\Omega_{\mathbf{m}}$. This notation is justified by the fact that the aforementioned canonical action of this invariant on $X_{\lambda}$ coincides with the endomorphism $\Omega_{\mathbf{m}}$ defined in the previous subsection. Thus, $\operatorname{Hom}\left(\mathbf{1}, \mathrm{C}\left[S_{t}\right]\right)=\operatorname{Span}\left(\left\{\Omega_{\mathbf{m}}\right\}\right)$ is a commutative algebra with basis $\Omega_{\mathbf{m}}$. It is easy to show that this algebra is the polynomial algebra $\mathrm{C}\left[Z_{1}, Z_{2}, \ldots\right]$ on the generators $Z_{j}=\Omega_{j, 0,0, \ldots}$, a fact exploited in CO.

2.7. A presentation of $\mathrm{C}\left[S_{t}\right]$ by generators and relations. It is well known that the group algebra $\mathrm{C}\left[S_{n}\right]$ with generators being simple reflections $s_{i j}$ is a quadratic algebra. Namely, the defining relations are:

$$
s_{i j}^{2}=1, s_{i j} s_{j k}=s_{i k} s_{i j}, s_{i j} s_{k l}=s_{k l} s_{i j},
$$

where different subscripts denote different indices. It is easy to interpolate this presentation to the case of complex rank, which would yield an inhomogeneous quadratic presentation of the algebra $\mathrm{C}\left[S_{t}\right]$, generated by $E=E_{1,0,0, \ldots}=\Delta_{2, t}^{S_{2}}$, valid at least for transcendental $t$.

One may consider the filtration on $\mathrm{C}\left[S_{t}\right]$ defined by the condition that $\operatorname{deg}(E)=1$, and the associated graded algebra $\operatorname{gr}\left(\mathrm{C}\left[S_{t}\right]\right)$. Similarly to the case of $S_{n}$ for integer $n$, this algebra is generated by the same generators with defining relations being the homogenization of the relations of C $\left[S_{t}\right]$. Indeed, for $S_{n}$ the homogenized relations look like

$$
s_{i j}^{2}=0, s_{i j} s_{j k}=s_{i k} s_{i j}, s_{i j} s_{k l}=s_{k l} s_{i j},
$$

and modulo these relations, any nonzero monomial in $s_{i j}$ may be rewritten as $s_{i_{1} j_{1}} \ldots s_{i_{m} j_{m}}$, so that the function $\max \left(i_{l}, j_{l}\right)$ strictly increases from left to right, and there are exactly $n$ ! such ordered monomials. This also shows that the ordered monomials are linearly independent,

\footnotetext{
${ }^{5}$ Note that the latter requirement is needed, as there are many $\mathbb{C}\left[S_{t}\right]$-modules in which the action of $\mathbb{C}\left[S_{t}\right]$ is different from the standard action of $\mathbb{C}\left[S_{t}\right]$ on the underlying object of $\operatorname{IndRep}\left(S_{t}\right)$, see Subsection 2.8 below.
} 
and that the quadratic algebra $\operatorname{gr}\left(\mathrm{C}\left[S_{n}\right]\right)$ is Koszul 6 (the quadratic Gröbner basis is formed by the unordered quadratic monomials, i.e. $s_{i j} s_{p q}$ with $\left.\max (i, j) \geq \max (p, q)\right)$. By interpolation, the same statements are true for $\mathrm{C}\left[S_{t}\right]$, at least for transcendental $t$.

Also, one can show that the Hilbert series of the algebra $\operatorname{gr}\left(\mathrm{C}\left[S_{t}\right]\right)$ is

$$
h(t, x)=x^{t} \frac{\Gamma\left(x^{-1}+t\right)}{\Gamma\left(x^{-1}\right)},
$$

where the function on the right should be replaced by its asymptotic expansion at $x \rightarrow+\infty$. Note that formal substitution of $x=1$ on the RHS ("order of $S_{t}$ ") gives $h_{t}(1)=\Gamma(1+t)$, which is $t$ ! for integer $t$, but this is illegitimate, as the series on the right side has zero radius of convergence, even though the algebra $\operatorname{gr}\left(\mathrm{C}\left[S_{t}\right]\right)$ is "finitely generated" (i.e. generated by an honest object, not just an ind-object, of $\operatorname{Rep}\left(S_{t}\right)$, namely the object $E$ ).

2.8. Modules over $\mathrm{C}\left[S_{t}\right]$. If $G$ is a finite group then the category of finite dimensional C $[G]$-modules in $\operatorname{Rep}(G)$ is equivalent the category $\boldsymbol{\operatorname { R e p }}(G \times G)=\boldsymbol{\operatorname { R e p }}(G) \otimes \boldsymbol{\operatorname { R e p }}(G)$. Indeed, given $X, Y \in \boldsymbol{\operatorname { R e p }}(G)$, we can take $X \otimes Y \in \boldsymbol{\operatorname { R e p }}(G)$ and introduce the action of $\mathrm{C}[G]$ on it by acting on the first tensor factor, which gives the desired equivalence. However, the category C $\left[S_{t}\right]$-fmod of finite dimensional C $\left[S_{t}\right]$-modules is not equivalent to $\operatorname{Rep}\left(S_{t}\right) \otimes \operatorname{Rep}\left(S_{t}\right)$. Indeed, C $\left[S_{t}\right]$-fmod contains a nontrivial invertible object $\mathbf{s}$ (of order 2), which we may call the sign representation, and which is $\mathbf{1}$ as an object of $\operatorname{Rep}\left(S_{t}\right)$, with the action of $E_{\mathbf{m}}$ defined by the map $(-1)^{\sum_{i} i m_{i}} \varepsilon$.

However, we have the following proposition.

Proposition 2.7. If $t$ is transcendental, then the category $\mathrm{C}\left[S_{t}\right]$-fmod is equivalent to $\operatorname{Rep}\left(S_{t}\right) \otimes \operatorname{Rep}\left(S_{t}\right) \oplus \operatorname{Rep}\left(S_{t}\right) \otimes \operatorname{Rep}\left(S_{t}\right) \otimes \mathbf{s}$ as a tensor category.

Proof. Let $N(\lambda)$ be a $\mathrm{Z}_{+}$-valued function on the set of all partitions which takes finitely many nonzero values. Let $X(N)=\oplus_{\lambda} N(\lambda) X_{\lambda}$ be the corresponding object of $\operatorname{Rep}\left(S_{t}\right)$. Our job is to show that for transcendental $t$, any action of $\mathrm{C}\left[S_{t}\right]$ on $X(N)$ comes from an object of $\operatorname{Rep}\left(S_{t}\right) \otimes \operatorname{Rep}\left(S_{t}\right) \oplus \operatorname{Rep}\left(S_{t}\right) \otimes \operatorname{Rep}\left(S_{t}\right) \otimes \mathbf{s}$. To this end, let us use the presentation of $\mathrm{C}\left[S_{t}\right]$ as a quotient of the tensor algebra $T E$

\footnotetext{
${ }^{6}$ I am grateful to Eric Rains for this remark.

${ }^{7}$ Note that such a thing clearly cannot happen for ordinary finitely generated graded algebras. Indeed, if $A$ is a graded algebra with generators $x_{1}, \ldots, x_{n}$ of degrees $d_{1}, \ldots, d_{n}$ then the radius of convergence of the Hilbert series $h_{A}(x)$ is bounded below by the real root of the equation $\sum_{i=1}^{n} x^{d_{i}}=1$ (since for the free algebra with such generators $h_{A}(t)=\left(1-\sum_{i=1}^{n} x^{d_{i}}\right)^{-1}$.
} 
by the appropriate relations, introduced above. This presentation implies that an action of $\mathrm{C}\left[S_{t}\right]$ on $X(N)$ is determined by a morphism $\rho \in \operatorname{Hom}_{S_{t}}(E \otimes X(N), X(N))$ satisfying certain quadratic relations. Now observe that the space $W:=\operatorname{Hom}_{S_{t}}(E \otimes X(N), X(N))$ is independent of $t$ (it has a basis given by certain diagrams in the partition algebra). Moreover, the quadratic relations for $\rho \in W$ depend polynomially on $t$. These relations define a family of closed subvarieties $Y_{t}$ in $W, t \in \mathrm{C}$ ("representation varieties"), with an action of the group $G L_{N}=G L_{N}(\mathrm{C}):=\prod_{\lambda} G L_{N(\lambda)}(\mathrm{C})$ by change of basis. This family is not necessarily flat; however, since the equations of $Y_{t}$ are polynomial in $t$ with rational coeffiicients, the set of $t$ for which $Y_{t}$ has a given finite number of $G L_{N}$-orbits is clearly semialgebraic, defined over $\mathrm{Q}$. So if we show that $\left|Y_{t} / G L_{N}\right|$ is a certain fixed number $m(N)$ for sufficiently large integer $t$, it will follow that $\left|Y_{t} / G L_{N}\right|=m(N)$ for any transcendental $t$.

To see that $\left|Y_{t} / G L_{N}\right|=m(N)$ for large integer $t=n$, note that, as explained above, $Y_{t}$ is the variety of representations of $S_{n} \times S_{n}$ which restrict to $X_{n}(N):=\oplus_{\lambda} N(\lambda) \pi_{\widetilde{\lambda}(n)}$ (whose interpolation is $X(N)$ ) on the diagonal subgroup $\left(S_{n}\right)_{\operatorname{diag}} \subset S_{n} \times S_{n}$. This variety clearly has finitely many orbits of $G L_{N}$, which implies the statement.

It remains to show that $m(N)$ equals the number of objects of $\operatorname{Rep}\left(S_{t}\right) \otimes \operatorname{Rep}\left(S_{t}\right) \oplus \operatorname{Rep}\left(S_{t}\right) \otimes \operatorname{Rep}\left(S_{t}\right) \otimes \mathbf{s}$ which map to $X(N)$ under the forgetful functor. To this end, we will use the following (well known) combinatorial lemma, whose proof is given in the appendix at the end of the paper.

Lemma 2.8. For each $C>0$ and $k \in \mathrm{Z}_{+}$there exists $N=N(C, k) \in$ $Z_{+}$such that for each $n \geq N$, if $V=\pi_{\mu}$ is an irreducible representation of $S_{n}$ such that $\operatorname{dim} V \leq C n^{k}$, then either the first row or the first column of $\mu$ has length $\geq n-k$.

Now let $n$ be large. Our job is to classify all ways to write $X_{n}(N)$ as $\oplus_{i=1}^{p} Y_{i} \otimes Y_{i}^{\prime}$, where $Y_{i}, Y_{i}^{\prime}$ are representations of $S_{n}$. Clearly, $p \leq$ $\sum_{\lambda} N(\lambda)$. Also, since $\operatorname{dim} X_{n}(N)$ is a polynomial of $n$ of some degree $k$, by Lemma 2.8, for large $n$ there are only finitely many possibilities for $Y_{i}$ and $Y_{i}^{\prime}$, and there are two options: either both have $\geq n-k$ boxes in the first row, or both have $\geq n-k$ boxes in the first column. This implies the required statement, and completes the proof of the proposition.

Remark 2.9. We expect that this proposition holds for all $t \notin Z_{+}$, but proving this would require a more refined approach. 
On the other hand, there are many infinite dimensional c $\left[S_{t}\right]$-modules (i.e. based on an ind-object of $\operatorname{Rep}\left(S_{t}\right)$ ) which are not ind-objects of $\operatorname{Rep}\left(S_{t}\right) \otimes \operatorname{Rep}\left(S_{t}\right)$. Indeed, there are only a countable collection of possible eigenvalues of the center of C $\left[S_{t}\right]$ on ind-objects of $\operatorname{Rep}\left(S_{t}\right) \otimes$ $\operatorname{Rep}\left(S_{t}\right)$.

The category C $\left[S_{t}\right]$-mod of (possibly infinite dimensional) C $\left[S_{t}\right]$-modules may be viewed as the category of "Harish-Chandra bimodules" for $S_{t}$ (as it is analogous to the category of Harish-Chandra bimodules for a semisimple Lie algebra). It would be interesting to study this category in more detail.

\section{Schur-Weyl duality For $\operatorname{Rep}\left(S_{t}\right)$}

In this section we discuss a Schur-Weyl duality for $\operatorname{Rep}\left(S_{t}\right)$, which generalizes the classical Schur-Weyl duality, and is based on the notion of a complex power of a vector space with a distinguished vector, in the case $t \notin \mathrm{Z}_{+}$. Under this duality, objects of $\operatorname{Rep}\left(S_{t}\right)$ correspond to objects of a parabolic category $\mathcal{O}$ for $\mathfrak{g l}_{n}$. This is discussed in much more detail and for general $t$ (including $t \in \mathrm{Z}_{+}$) in the forthcoming paper EA2].

\subsection{Unital vector spaces.}

Definition 3.1. A unital vector space is a vector space $V$ which has a distinguished nonzero vector 1 .

It is clear that unital vector spaces form a symmetric monoidal category under tensor product.

Let $(V, 1)$ be a unital vector space, and let $\bar{V}:=V / \mathrm{C} 1$. Fix a splitting $\bar{V} \rightarrow V$, and denote its image by $U$; thus, we have $V=\mathrm{C} 1 \oplus U$. This gives an isomorphism $\operatorname{Aut}(V, 1) \cong G L(U) \ltimes U^{*}$.

For a partition $\lambda$, let $S^{\lambda}$ be the corresponding Schur functor on the category of vector spaces. Define the induced representation of $\operatorname{Aut}(V, 1)$ given by the formula

$$
E_{\lambda}:=\operatorname{Ind}_{G L(U)}^{G L(U) \ltimes U^{*}} \mathrm{~S}^{\lambda} U
$$

i.e., $E_{\lambda}=S U \otimes S^{\lambda} U$, with the obvious action of $G L(U)$, and the action of $U^{*}$ given by differentiation in the first component. Note that the action of the Lie algebra $\operatorname{Lie}(\operatorname{Aut}(V, 1))=\mathfrak{g l}(U) \ltimes U^{*}$ on $E_{\lambda}$ naturally extends to an action of the Lie algebra $\mathfrak{g l}(V)$; indeed, we can write $E_{\lambda}$ as

$$
E_{\lambda}=\operatorname{Hom}_{\mathfrak{g l}(U) \ltimes U}^{\mathrm{res}}\left(U(\mathfrak{g l}(V)), \mathrm{S}^{\lambda} U\right),
$$

where $U$ acts on $S^{\lambda} U$ by zero, and the superscript "res" means that we are taking the restricted space of homomorphisms with respect to the 
grading in which $\operatorname{deg}(1)=0, \operatorname{deg}(U)=1$ (i.e., the space spanned by homogeneous homomorphisms). Thus, $E_{\lambda}$ is a module for the HarishChandra pair $(\mathfrak{g l}(V), \operatorname{Aut}(V, 1))$.

Proposition 3.2. (i) The module $E_{\lambda}$ is the contragredient module $M(t-|\lambda|, \lambda)^{\vee}$ to the the parabolic Verma module $M(t-|\lambda|, \lambda)$ over $\mathfrak{g l}(V)$ with highest weight $(t-|\lambda|, \lambda)$ (integrating to the subgroup $\operatorname{Aut}(V, 1))$.

(ii) If $t \notin \mathrm{Z}_{+}$, the module $E_{\lambda}$ is irreducible, and hence is isomorphic to the Verma module $M(t-|\lambda|, \lambda)$.

Proof. Let $u$ be a highest weight vector of $\mathrm{S}^{\lambda} U$ as a $\mathfrak{g l}(U)$-module (clearly, it has weight $\lambda$ ). Then $u$ is a highest weight vector for $\mathfrak{g l}(V)$ of weight $(t-|\lambda|, \lambda)$, since Id $\circ u=t u$. So we have a natural homomorphism $M(t-|\lambda|, \lambda) \rightarrow E_{\lambda}$ sending the highest weight vector of $M(t-|\lambda|, \lambda)$ to $u$, which implies (i).

The characters of the two modules are the same, so to prove (ii), it suffices to show that for $t \notin \mathrm{Z}_{+}$, the module $M(t-|\lambda|, \lambda)$ is irreducible.

Assume that $M(t-|\lambda|, \lambda)$ is reducible. Then it must contain a singular vector of weight $(t-|\lambda|, \lambda)-m \alpha$, where $\alpha$ is a positive root, and $m$ a positive integer (this follows, for instance, from the Jantzen determinant formula for parabolic Verma modules). Then, setting $N=$ $\operatorname{dim} V$ and $\rho=(N, N-1, \ldots, 1)$, we must have

$$
(t-|\lambda|, \lambda)+\rho-m \alpha=s_{\alpha}((t-|\lambda|, \lambda)+\rho) .
$$

Since the submodule generated by the singular vector integrates to $\operatorname{Aut}(V, 1)$, this implies that all the coordinates of the vector

$$
s_{\alpha}((t-|\lambda|, \lambda)+\rho)
$$

except the first one form a strictly decreasing sequence. Thus, $\alpha=$ $e_{1}-e_{i}$ for some $i$.

Now let $\lambda$ be a partition with at most $\operatorname{dim} V-1$ parts and $n \geq \lambda_{1}+|\lambda|$. If the Verma module $M(t-|\lambda|, \lambda)$ has a submodule with highest weight vector of weight $(t-|\lambda|, \lambda)-m\left(e_{1}-e_{i}\right)$, then we must have

$$
t-|\lambda|-m+N=\lambda_{i}+N-i
$$

i.e.,

$$
t=|\lambda|+\lambda_{i}+m-i .
$$

Thus, $t$ is an integer. Moreover, $\lambda_{i-1} \geq \lambda_{i}+m \geq m$, so $|\lambda| \geq(i-1) m$, SO

$$
t \geq(i-1) m+m-i=i(m-1) \geq 0 .
$$

So $t \in Z_{+}$, as desired. 
Proposition 3.3. There is a unique, up to scaling, nonzero $\operatorname{Aut}(V, 1)$ homomorphism $f_{\lambda, n}: S^{\tilde{\lambda}(n)} V \rightarrow E_{\lambda}$, and this homomorphism is injective.

Proof. By Frobenius reciprocity

$$
\operatorname{Hom}_{\operatorname{Aut}(V, 1)}\left(\mathrm{S}^{\widetilde{\lambda}(n)} V, E_{\lambda}\right)=\operatorname{Hom}_{G L(U)}\left(\mathrm{S}^{\widetilde{\lambda}(n)}(U \oplus \mathrm{C}), \mathrm{S}^{\lambda} U\right) \text {. }
$$

According to the branching rules for general linear groups, this space is 1-dimensional. Thus, there is a unique, up to scaling, nonzero $\operatorname{Aut}(V, 1)$-homomorphism $f_{\lambda, n}: S^{\widetilde{\lambda}(n)} V \rightarrow E_{\lambda}$.

Let us show that $f_{\lambda, n}$ are injective. Assume the contrary, and let $0 \neq y \in \mathrm{S}^{\widetilde{\lambda}(n)} V$ be a vector such that $f_{\lambda, n}(y)=0$. It is easy to show that by applying elements of $\mathfrak{g l}(U) \ltimes U^{*}$ to $y$, we can map $y$ to a nonzero vector $y^{\prime} \in \mathrm{S}^{\lambda} U \subset \mathrm{S}^{\widetilde{\lambda}(n)} V$ such that $f_{\lambda, n}\left(y^{\prime}\right)=0$. But this is a contradiction, since $\left.f_{\lambda, n}\right|_{S^{\lambda} U}$ is clearly injective.

We will normalize $f_{\lambda, n}$ so that it corresponds to the canonical element in $\operatorname{Hom}_{G L(U)}\left(\mathrm{S}^{\widetilde{\lambda}(n)}(U \oplus \mathrm{C}), \mathrm{S}^{\lambda} U\right)$.

Now consider the natural homomorphism $\phi_{\lambda, n}: \mathrm{S}^{\widetilde{\lambda}(n)} V \hookrightarrow \mathrm{S}^{\widetilde{\lambda}(n+1)} V$, defined by setting the second argument of the natural projection

$$
\mathrm{S}^{\widetilde{\lambda}(n)} V \otimes V \rightarrow \mathrm{S}^{\widetilde{\lambda}(n+1)} V
$$

to be 1. It is easy to show that this homomorphism is nonzero.

Proposition 3.4. The homomorphism $\phi_{\lambda, n}$ is injective.

Proof. By Proposition 3.3, the homomorphisms $f_{\lambda, n}$ are compatible with the homomorphisms $\phi_{\lambda, n}: S^{\widetilde{\lambda}(n)} V \hookrightarrow S^{\widetilde{\lambda}(n+1)} V$, i.e. $f_{n+1, \lambda} \circ \phi_{\lambda, n}=$ $f_{\lambda, n}$. This implies that $\phi_{\lambda, n}$ are injective, as desired.

Now let $\mathrm{S}^{\lambda, \infty} V$ be the direct limit of $\mathrm{S}^{\tilde{\lambda}(n)} V$ with respect to the inclusions $\phi_{\lambda, n}: \mathrm{S}^{\lambda, \infty} V=\lim _{n \rightarrow \infty} \widetilde{S}^{\widetilde{\lambda}(n)} V$.

Proposition 3.5. Let $V=\mathrm{C} 1 \oplus U$ be a splitting. Then there is a canonical isomorphism of the representation $\mathrm{S}^{\lambda, \infty} V$ with the induced representation $E_{\lambda}$.

This proposition shows that the representation $E_{\lambda}$ in fact does not depend on the splitting of $V$, and is functorially attached to $(V, 1)$.

Proof. By Propositions 3.313.4, we have an embedding $f_{\lambda, \infty}=\lim _{n \rightarrow \infty} f_{\lambda, n}$ : $\mathrm{S}^{\lambda, \infty} V \rightarrow S U \otimes \mathrm{S}^{\lambda} U$. Comparing the restrictions of both modules to $G L(U)$ using branching rules, we see that this embedding must be an isomorphism. Indeed, both $G L(U)$-modules are multiplicity free, and are direct sums of the irreducible modules $S^{\mu} U$, where $\mu$ runs over 
partitions with at most $\operatorname{dim} V-1$ parts such that $\mu_{i} \geq \lambda_{i} \geq \mu_{i+1}$ for $i \geq 1$.

3.2. Complex powers of a unital vector space. Let $x$ be a variable. If $t$ is an arbitrary complex number, then the function $x^{t}$ does not have an algebraic meaning. On the other hand, the function $(1+x)^{t}$ does: it is just the formal power series

$$
(1+x)^{t}=\sum_{m=0}^{\infty} \frac{t(t-1) \ldots(t-n+1)}{n !} x^{n} .
$$

Similarly, if $V$ is a vector space, then we cannot naturally define $V^{\otimes t}$ for arbitrary complex $t$, but we can do so if $V$ is a unital vector space.

Definition 3.6. Let $(V, 1)$ be a unital vector space. For $t \notin z_{+}$define $V^{\otimes t}$ to be the ind-object of the category $\operatorname{Rep}\left(S_{t}\right)$ given by the formula

$$
V^{\otimes t}=\oplus_{\lambda} S^{\lambda, \infty} V \otimes X_{\lambda} .
$$

This is clearly an interpolation of $V^{\otimes n}=\oplus_{\lambda} \mathrm{S}^{\widetilde{\lambda}(n)} V \otimes \pi_{\tilde{\lambda}(n)}$ to complex rank.

Thus we have a complex rank analog of the Schur-Weyl functor, $S W_{t}: \operatorname{Rep}\left(S_{t}\right)^{\text {op }} \rightarrow \operatorname{Ind} \operatorname{Rep}(\operatorname{Aut}(V, 1))$, which is given by the formula

$$
S W_{t}(\pi)=\operatorname{Hom}\left(\pi, V^{\otimes t}\right),
$$

and $S W_{t}\left(X_{\lambda}\right)=E_{\lambda}$ for all partitions $\lambda$ (note that this is zero if $\lambda$ has more than $\operatorname{dim} V-1$ parts).

Proposition 3.7. Assume that $(V, 1)$ is a nonnegatively graded (or filtered) unital vector space with $V[0]$ spanned by 1 . Let the Hilbert series of $V$ be $h_{V}(x)=1+O(x)$. Then the Hilbert series of $V^{\otimes t}$ is $h(x)^{t}$.

Proof. The proof is by interpolation from integer $n$.

Let $\operatorname{gr} V:=\mathrm{C} 1 \oplus \bar{V}$ be the associated graded of the space $V$ with respect to its natural 2-step filtration. The object $V^{\otimes t}$ has a natural ascending $\mathrm{Z}_{+}$-filtration such that $\operatorname{gr}\left(V^{\otimes t}\right)=(\operatorname{gr} V)^{\otimes t}$. Namely, for each Young diagram $\mu$ we have an ascending filtration on $\mathrm{S}^{\mu} V$, which is induced by the filtration on $V$; this filtration is compatible with the inclusions $\phi_{n, \lambda}: \mathrm{S}^{\widetilde{\lambda}(n)} V \hookrightarrow \mathrm{S}^{\widetilde{\lambda}(n+1)} V$ and thus defines a filtration on $\mathrm{S}^{\lambda, \infty} V$. This gives rise to a filtration on $V^{\otimes t}$ by taking direct sum.

Proposition 3.8. One has

$$
\operatorname{gr} V^{\otimes t} \cong S \bar{V} \otimes\left(\oplus_{\lambda} \mathrm{S}^{\lambda} \bar{V} \otimes X_{\lambda}\right) .
$$

Proof. This follows immediately from Proposition 3.5. 
Proposition 3.9. One has

$$
F_{0} V^{\otimes t}=\mathbf{1}, F_{1} V^{\otimes t}=(V \otimes \mathfrak{h}) / \mathfrak{h}_{0}=V \otimes \mathbf{1} \oplus \bar{V} \otimes \mathfrak{h}_{0} .
$$

Proof. The first statement is obvious. To prove the second statement, note that $\lambda=\emptyset$ and $\lambda=(1)$ are the only partitions contributing to $F_{1}$ (this is easily seen by looking at the associated graded object). Now, the contribution of $\lambda=\emptyset$ is $V$, and the contribution of $\mathfrak{h}_{0}=X_{(1)}$ is $\bar{V} \otimes \mathfrak{h}_{0}$, as desired.

Proposition 3.10. The assignment $V \mapsto V^{\otimes t}$ is a (non-additive) symmetric monoidal functor from the category of unital vector spaces to $\operatorname{Ind} \operatorname{Rep}\left(S_{t}\right)$.

Proof. Let $V, W$ be two unital vector spaces. For every nonnegative integer $n$ we have morphisms

$$
J_{n}: V^{\otimes n} \otimes W^{\otimes n} \rightarrow(V \otimes W)^{\otimes n} .
$$

and $J_{n}^{\prime}=J_{n}^{-1}$. These morphisms are polynomial in $n$ in an appropriate sense, hence they interpolate to morphisms $J: V^{\otimes t} \otimes W^{\otimes t} \rightarrow(V \otimes W)^{\otimes t}$ and $J^{\prime}:(V \otimes W)^{\otimes t} \rightarrow V^{\otimes t} \otimes W^{\otimes t}$, such that $J \circ J^{\prime}=1, J^{\prime} \circ J=1$. Also, $J$ is a symmetric monoidal structure, since it is one for integer $t$.

Let $\operatorname{Res}_{S_{t-1}}^{S_{t}}: \operatorname{Rep}\left(S_{t}\right) \rightarrow \operatorname{Rep}\left(S_{t-1}\right)$ be the restriction functor.

Proposition 3.11. We have a natural isomorphism $\psi_{t}: V^{\otimes t-1} \otimes V \rightarrow$ $\operatorname{Res}_{S_{t-1}}^{S_{t}} V^{\otimes t}$, which commutes with the action of $\mathfrak{g l}(V)$.

Proof. The morphism $\psi_{t}$ is constructed by interpolation from integer $t$, and it is easy to see that it is an isomorphism (this follows from the decomposition of $V \otimes M_{(t-1-|\lambda|, \lambda)}$ into irreducible $\mathfrak{g l}(V)$-modules).

\section{WREATH PRODUCTS}

In this section we use the notion of a complex power of a unital vector space to construct wreath products of complex rank (for any associative algebra $A$ ). In the case when $A$ is a group algebra, this was done in a different way by Knop, [Kn1, Kn2].

4.1. Complex powers of a unital algebra. Now assume that $V=A$ is a unital associative algebra with unit 1 . Then, by Proposition 3.10 , $A^{\otimes t}$ is also an algebra (in $\operatorname{IndRep}\left(S_{t}\right)$ ). This algebra interpolates the algebra $A^{\otimes n}$ in $\operatorname{Ind} \operatorname{Rep}\left(S_{n}\right)$, whose category of modules in $\operatorname{Ind} \operatorname{Rep}\left(S_{n}\right)$ is naturally equivalent to the category of modules over the wreath product C $\left[S_{n}\right] \ltimes A^{\otimes n}$. Thus we can think of the category of modules over $A^{\otimes t}$ in $\operatorname{IndRep}\left(S_{t}\right)$ as an interpolation to complex rank of the category 
of modules over the wreath product C $S_{n} \ltimes A^{\otimes n}$; it will thus be denoted by $\widehat{\operatorname{Rep}}\left(S_{t} \ltimes A^{\otimes t}\right)$ (the hat is used to emphasize that the modules are allowed to be infinite dimensional, i.e. ind-objects of $\operatorname{Rep}\left(S_{t}\right)$ ).

Similarly to the case $A=\mathrm{C}$ considered above, we can define the wreath product algebra C $\left[S_{t}\right] \ltimes A^{\otimes t}$ in $\operatorname{IndRep}\left(S_{t}\right)$. Namely, the Hopf algebra C $\left[S_{t}\right]$ acts naturally on $A^{\otimes t}\left(\right.$ since $A^{\otimes t}$ is an algebra in $\operatorname{IndRep}\left(S_{t}\right)$ ), so C $\left[S_{t}\right] \ltimes A^{\otimes t}$ is defined just as the usual smash product from the theory of Hopf actions, formed inside $\operatorname{IndRep}\left(S_{t}\right)$. Moreover, $A^{\otimes t}$-modules is the same thing as C $\left[S_{t}\right] \ltimes A^{\otimes t}$-modules, in which the subalgebra C $\left[S_{t}\right]$ acts via its canonical action (see Remark 2.5).

Note also that if $A$ is a Hopf algebra, then so are $A^{\otimes t}$ and C $\left[S_{t}\right] \ltimes A^{\otimes t}$. Hence, in this case, the category of $A^{\otimes t}$-modules is a tensor category (see Remark 2.5). 8

Let us study the structure of $A^{\otimes t}$ in more detail. Define the ordinary algebra $S^{t} A:=\operatorname{Hom}\left(\mathbf{1}, A^{\otimes t}\right)$ (in the category of vector spaces).

Proposition 4.1. For $t \notin \mathrm{Z}_{+}$the algebra $S^{t} A:=\operatorname{Hom}\left(\mathbf{1}, A^{\otimes t}\right)$ is naturally isomorphic to $U(A) /(1=t)$, where $U(A)$ is the universal enveloping algebra of $A$ regarded as a Lie algebra.

Proof. We have a natural linear map $A \rightarrow S^{t} A \hookrightarrow A^{\otimes t}$ given as the composition $A \rightarrow A \otimes \mathfrak{h} \rightarrow F_{1} A^{\otimes t}$, where the first map is $1 \otimes \iota, \iota$ being the unit map of the algebra $\mathfrak{h}$, and the second map is the map of Proposition 3.9. It is easy to check that this map is a Lie algebra homomorphism, such that $1 \rightarrow t$, so it gives rise to a homomorphism of associative algebras $\zeta_{t}: U(A) /(1-t) \rightarrow S^{t} A$. This homomorphism interpolates the natural homomorphism $\zeta_{n}: U(A) /(1=n) \rightarrow S^{n} A$ for nonnegative integer $n$, given by

$$
a \rightarrow a_{1}+\ldots+a_{n}, a \in A,
$$

where $a_{i}=1^{\otimes i-1} \otimes a \otimes 1^{n-i} \in A^{\otimes n}$.

To check that $\zeta_{t}$ is an isomorphism for $t \notin \mathrm{Z}_{+}$, consider the filtration inn $A$ defined by $\mathcal{F}_{0} A=\mathrm{C} 1, \mathcal{F}_{1}(A)=A$. Then $\operatorname{gr}(A)$ is the commutative algebra $\mathrm{C} 1 \oplus U$, where $U=A / \mathrm{C} 1$, and the product of any two elements of $U$ is zero. The filtration $\mathcal{F}$ extends naturally to $U(A)$ and to $S^{t} A$, and is preserved by the map $\zeta_{t}$. Thus, it suffices to check that $\operatorname{gr} \zeta_{t}$ is an isomorphism. We have $\operatorname{gr}(U(A) /(1-t)) \cong S \operatorname{gr}(A) /(1-t) \cong S U$, and by the results of the previous section, $\operatorname{gr}\left(S^{t} A\right) \cong S^{t} \operatorname{gr}(A) \cong S U$.

\footnotetext{
${ }^{8}$ Note that this is slight abuse of terminology, since in this category, only finite dimensional objects (i.e., those which are honest objects of $\operatorname{Rep}\left(S_{t}\right)$, rather than ind-objects) are rigid.
} 
After these identifications, it is easy to see that $\operatorname{gr}\left(\zeta_{t}\right)$ becomes the identity map. This implies the statement.9

Corollary 4.2. Let $M$ be a left $A^{\otimes t}$-module in $\operatorname{Ind} \operatorname{Rep}\left(S_{t}\right)$. Let $\pi \in$ $\operatorname{Rep}\left(S_{t}\right)$. Then $\operatorname{Hom}(\pi, M)$ is naturally a representation of the Lie algebra $A$ with 1 acting by multiplicaton by $t$.

Proof. This follows immediately from Proposition 4.1, since $\operatorname{Hom}\left(\mathbf{1}, A^{\otimes t}\right)$ acts on $\operatorname{Hom}(\pi, M)$ for any $\pi$.

Now we would like to describe the algebra $A^{\otimes t}$ by generators and relations. In the classical case, the tensor power algebra $A^{\otimes n}$ can be presented as follows. The generators are $A \otimes \mathrm{C}^{n}$ (spanned by $\left.a_{1}, \ldots, a_{n}, a \in A\right)$, and the defining relations are:

$$
1_{i}=1_{j}, i \neq j ; a_{i} b_{i}=(a b)_{i} ; a_{i} b_{j}=b_{j} a_{i}, i \neq j, \text { for } a, b \in A .
$$

By analogy, in the complex rank case, as generators we will take $F_{1} A^{\otimes t}$, which, by Proposition 3.9, is $A \otimes \mathfrak{h} / \mathfrak{h}_{0}$. More precisely, we will use $A \otimes \mathfrak{h}$ as generators, and include $\mathfrak{h}_{0}=\mathrm{C} \cdot 1 \otimes \mathfrak{h}_{0} \subset A \otimes \mathfrak{h}$ in the ideal of relations, which incorporates the first relation in (2).

There are two other relations among the generators in $A \otimes \mathfrak{h}$, which interpolate the second and the third relation in (2), respectively. To write them, let $m: \mathfrak{h} \otimes \mathfrak{h} \rightarrow \mathfrak{h}$ be the natural commutative product, and $m^{*}: \mathfrak{h} \rightarrow \mathfrak{h} \otimes \mathfrak{h}$ be the dual map to $m$. Also let $\mu_{A}$ be the multiplication in $A$, and $[,]_{A}$ the commutator in $A$. The first relation is the image of the morphism $\xi: A \otimes A \otimes \mathfrak{h} \rightarrow(A \otimes \mathfrak{h}) \oplus(A \otimes \mathfrak{h})^{\otimes 2}$ given by

$$
\xi=\mu_{A} \otimes \mathrm{Id}-\mathrm{Id} \otimes \mathrm{Id} \otimes m^{*} .
$$

The second relation is the image of the morphism $\eta: A \otimes \mathfrak{h} \otimes A \otimes \mathfrak{h} \rightarrow$ $(A \otimes \mathfrak{h}) \oplus(A \otimes \mathfrak{h})^{\otimes 2}$ given by the formula

$$
\eta=\mathrm{Id}-\sigma_{13} \sigma_{24}-\left([,]_{A} \otimes m\right) \circ \sigma_{23},
$$

where $\sigma_{i j}$ denotes the permutation of the $i$-th and the $j$-th factor.

Proposition 4.3. The algebra $A^{\otimes t}$ is the quotient of $T(A \otimes \mathfrak{h})$ by the ideal I generated by $\mathfrak{h}_{0} \subset A \otimes \mathfrak{h}, \operatorname{Im} \xi$, and $\operatorname{Im} \eta$.

Proof. It is easy to see that we have a natural homomorphism

$$
\theta_{A}: T(A \otimes \mathfrak{h}) / I \rightarrow A^{\otimes t}
$$

(interpolating the case of integer $t$ ). This homomorphism preserves natural filtrations, and $\operatorname{gr}\left(\theta_{A}\right)=\theta_{\operatorname{gr} A}$, where $\operatorname{gr} A=\mathrm{C} 1 \oplus \bar{A}, \bar{A}:=A / \mathrm{C} 1$,

\footnotetext{
${ }^{9}$ The fact that $\zeta_{t}$ is an isomorphism for generic $t$ also follows from the fact that $\zeta_{n}$ is surjective for each $n$, and asymptotically injective when $n \rightarrow \infty$ (i.e., injective in any fixed filtration degree for sufficiently large $n$ ).
} 
with multiplication on $\bar{A}$ being zero. It is easy to check that $\theta_{\text {gr } A}$ is an isomorphism, which implies that so is $\theta_{A}$.

Remark 4.4. In a similar way, one can define the algebra C $\left[S_{t}\right] \ltimes A^{\otimes t}$ inside IndRep $\left(S_{t}\right)$ by generators and relations. Namely, in the classical setting, combining relations (11) and (2), we see that the algebra C $\left[S_{n}\right] \ltimes$ $A^{\otimes n}$ is generated by $a_{i}, a \in A$, and $s_{i j}$, with the relations

$$
\begin{gathered}
s_{i j}^{2}=1, s_{i j} s_{j k}=s_{i k} s_{i j}, s_{i j} s_{k l}=s_{k l} s_{i j}, \\
1_{i}=1_{j}, i \neq j ; a_{i} b_{i}=(a b)_{i} ; a_{i} b_{j}=b_{j} a_{i}, i \neq j, \text { for } a, b \in A, \\
s_{i j} a_{i}=a_{j} s_{i j},
\end{gathered}
$$

where different subscripts represent different indices. These relations are easy to interpolate to complex rank, similarly to how one does it for (11) and (2) separately, and one defines the algebra C $\left[S_{t}\right] \ltimes A^{\otimes t}$ as the quotient of $T(E \oplus A \otimes \mathfrak{h})$ by the interpolation of these relations.

Remark 4.5. Proposition 4.3 provides a construction of the complex tensor power $V^{\otimes t}$ for $V=\mathrm{C} 1 \oplus U$, which does not use representation theory of $\mathfrak{g l}(V)$. Namely, define a unital algebra structure on $V$ by declaring 1 a unit and setting $u_{1} u_{2}=0$ for $u_{1}, u_{2} \in U$. Then we can define $V^{\otimes t}$ to be the algebra defined by the presentation of Proposition 4.3 .

Remark 4.6. It is easy to see that if $V$ is an $A$-module then $V^{\otimes t}$ is naturally an $A^{\otimes t}$-module (even if $\mathrm{C} 1 \subset V$ is not fixed by $A$ ). Namely, the action of the generators $A \otimes \mathfrak{h}$ on $V^{\otimes t}$ is obtained by interpolating from integer $t$. Thus for every $A$-module $V$ we get a module $M_{t}(V, v):=$ $V^{\otimes t}$ (with distinguished vector $v$ ) over $A^{\otimes t}$ for any choice of a nonzero vector $1=v \in V$ (clearly, this vector matters only up to scaling). In particular, by taking invariants, it defines a module $\operatorname{Hom}\left(\mathbf{1}, M_{t}(V, v)\right)=$ $S^{t} V$ over the Lie algebra $A$ with 1 acting by multiplication by $t$. For example, as explained in the previous section, if $A=\operatorname{End} V$ and $V$ is finite dimensional, then $\operatorname{Hom}\left(\mathbf{1}, M_{t}(V, v)\right)$ is the parabolic Verma module over End $V=\mathfrak{g l}(V)$ with highest weight $(t, 0,0, \ldots)$ integrable to $\operatorname{Aut}(V, v)$. Note that this module depends in an essential way on the choice of the line in $V$ generated by $v$ (and hence so does $M_{t}(V, v)$ ).

4.2. Knop's category $\operatorname{Rep}\left(S_{t} \ltimes \Gamma^{t}\right)$. Let $\Gamma$ be a finite group. Recall that the irreducible representations of the wreath product $S_{n} \ltimes \Gamma^{n}$ are labeled by maps

$$
\lambda: \text { Irrep } \Gamma \rightarrow \text { Partitions, }
$$


$V \rightarrow \lambda_{V}$, such that $\sum_{V}\left|\lambda_{V}\right|=n$. Namely, the representation corresponding to $\lambda$ is

$$
\pi_{\lambda}=\operatorname{Ind}_{\prod_{V} S_{\left|\lambda_{V}\right|} \ltimes \Gamma^{n}}^{S_{n} \Gamma_{V}^{n}} \bigotimes_{V}\left(\pi_{\lambda_{V}} \otimes V^{\otimes\left|\lambda_{V}\right|}\right) .
$$

By analogy with Deligne's construction, F. Knop constructed a Karoubian tensor category $\operatorname{Rep}\left(S_{t} \ltimes \Gamma^{t}\right)$ for complex $t$, interpolating the categories $\boldsymbol{\operatorname { R e p }}\left(S_{n} \ltimes \Gamma^{n}\right)([\mathrm{Kn} 2])$. More precisely, if $t$ is a nonnegative integer, then the category $\operatorname{Rep}\left(S_{t} \ltimes \Gamma^{t}\right)$ projects onto the category $\operatorname{Rep}\left(S_{n} \ltimes \Gamma^{n}\right)$.

The indecomposable objects $X_{\lambda}$ of $\operatorname{Rep}\left(S_{t} \ltimes \Gamma^{t}\right)$ are labeled by all maps

$$
\lambda: \text { Irrep } \Gamma \rightarrow \text { Partitions, }
$$

$V \rightarrow \lambda_{V}$. Similarly the case of $\Gamma=1$, the objects $X_{\lambda}$ interpolate the representations $\pi_{\widetilde{\lambda}(n)}$, where $\sum_{V} \widetilde{\lambda}(n)_{V}=n$, and $\widetilde{\lambda}(n)_{V}=\lambda_{V}$ for $V \neq \mathrm{C}$, while

$$
\widetilde{\lambda}(n)_{\mathrm{C}}=\left(n-|\lambda|,\left(\lambda_{\mathrm{C}}\right)_{1},\left(\lambda_{\mathrm{C}}\right)_{2}, \ldots\right) .
$$

It is easy to check that the tensor category $\operatorname{Rep}\left(S_{t} \ltimes \Gamma^{t}\right)$ is a full subcategory of the category of modules over the Hopf algebra $\mathrm{C}[\Gamma]^{\otimes t}$.

Remark 4.7. For any tensor category $\mathcal{C}$, M. Mori in [Mo] defined a new tensor category $S_{t}(\mathcal{C})$, such that if $\mathcal{C}=\operatorname{Rep}(\Gamma)$ then $S_{t}(\mathcal{C})=$ $\operatorname{Rep}\left(S_{t} \ltimes \Gamma^{t}\right)$. If $\mathcal{C}=H$-fmod (finite dimensional modules), where $H$ is a Hopf algebra then $S_{t}(\mathcal{C})$ is a full subcategory of $H^{\otimes t}$-mod.

4.3. The central elements for wreath products. Every conjugacy class of the wreath product $S_{n} \ltimes \Gamma^{n}$ gives rise to a central element in its group algebra (the sum of all elements in the conjugacy class). For the class of transpositions, we will denote this central element just by $\Omega$. For the class defined by a nontrivial conjugacy class $C \subset \Gamma$, we'll denote the central element by $\Omega_{C}$.

Similarly to the case of $S_{n}$, the elements $\Omega$ and $\Omega_{C}$ can be interpolated to complex $t$ (to define endomorphisms of the identity functor of $\left.\operatorname{Rep}\left(S_{t} \ltimes \Gamma^{t}\right)\right)$. If $t \notin \mathrm{Z}_{+}$, this can be done directly by interpolation from large integer $n$. Namely, it is easy to check that $\Omega$ and $\Omega_{C}$ act on $\pi_{\widetilde{\lambda}(n)}$ by scalars which are polynomials in $n$, so we can define them for $t \notin \mathrm{z}_{+}$on $X_{\lambda}$ by substituting $t$ instead of $n$ into these polynomials.

\section{Interpolation of Degenerate AFFine HeCKe Algebras AND SYMPLECTIC REFLECTION ALGEBRAS}

5.1. Interpolation of degenerate affine Hecke algebras of type

A. Let $\mathfrak{h}=\mathfrak{h}_{0} \oplus \mathrm{C}$ be the permutation representation of the symmetric 
group $S_{n}, n \geq 1$. Let $\Sigma$ be the set of reflections (i.e., transpositions) in $S_{n}$.

Recall $([\mathrm{Dr}])$ that the degenerate affine Hecke algebra (dAHA) $\mathcal{H}_{k}(n)$ of type $A$ (for $k \in \mathrm{C}$ ) is the quotient of $\mathrm{C}\left[S_{n}\right] \ltimes T \mathfrak{h}$ by the relations

$$
\left[y, y^{\prime}\right]=k \sum_{s, s^{\prime} \in \Sigma}\left(s y, s^{\prime} y^{\prime}\right)_{\mathfrak{h}}\left[s, s^{\prime}\right], y, y^{\prime} \in \mathfrak{h} .
$$

where $(,)_{\mathfrak{h}}$ is the natural inner product on $\mathfrak{h}$.

This implies that modules over $\mathcal{H}_{k}(n)$ can be described categorically as follows.

Proposition 5.1. An $\mathcal{H}_{k}(n)$-module is the same thing as an $S_{n}$-module $M$ equipped with an $S_{n}$-morphism

$$
y: \mathfrak{h} \otimes M \rightarrow M
$$

such that the morphism

$$
y \circ(1 \otimes y) \circ((1-\sigma) \otimes 1): \mathfrak{h} \otimes \mathfrak{h} \otimes M \rightarrow M,
$$

(where $\sigma$ is the permutation of components) equals

$$
k\left((,)_{\mathfrak{h}} \otimes 1\right) \circ\left[\Omega^{13}, \Omega^{23}\right],
$$

where $\Omega_{i j}$ is the Jucys-Murphy morphism $\Omega$ acting in the tensor product of the $i$-th and the $j$-th factor.

The object $\mathfrak{h}$ (the permutation representation) is defined in the category $\operatorname{Rep}\left(S_{t}\right)$ for any $t$, and has a natural symmetric pairing $(,)_{\mathfrak{h}}$ : $\mathfrak{h} \otimes \mathfrak{h} \rightarrow \mathbf{1}$. Therefore, Proposition 5.1 allows us to define the interpolation of the category of modules over $\mathcal{H}_{k}(n)$. Namely, we make the following

Definition 5.2. Let $t \in \mathrm{C}$. An object of the category $\widehat{\operatorname{Rep}}\left(\mathcal{H}_{k}(t)\right)$ is an ind-object of Deligne's category $\operatorname{Rep}\left(S_{t}\right)$ equipped with a morphism

$$
y: \mathfrak{h} \otimes M \rightarrow M
$$

such that the morphism

$$
y \circ(1 \otimes y) \circ((1-\sigma) \otimes 1): \mathfrak{h} \otimes \mathfrak{h} \otimes M \rightarrow M,
$$

equals

$$
k\left((,)_{\mathfrak{h}} \otimes 1\right) \circ\left[\Omega^{13}, \Omega^{23}\right] .
$$

Morphisms in $\widehat{\operatorname{Rep}}\left(\mathcal{H}_{k}(t)\right)$ are morphisms in $\operatorname{IndRep}\left(S_{t}\right)$ which respect $y$.

Remark 5.3. Note that similarly to the classical case, $k$ can be rescaled without changing the category (so it can be made 0 or 1 ). 
Remark 5.4. Alternatively, we can define the algebra $\mathcal{H}_{k}(t)$ in $\operatorname{Rep}\left(S_{t}\right)$ to be the quotient of $\mathrm{C}\left[S_{t}\right] \ltimes T \mathfrak{h}$ by the interpolation of the defining relation of $\mathcal{H}_{k}(n)$. Then the category $\widehat{\operatorname{Rep}}\left(\mathcal{H}_{k}(t)\right)$ may be defined as the category of modules over $\mathcal{H}_{k}(t)$ in $\operatorname{Rep}\left(S_{t}\right)$ in which C $\left[S_{t}\right]$ acts by the canonical action. A similar remark applies to the examples below (degenerate AHA of type B and wreath product symplectic reflection algebras, in particular, rational Cherednik algebras).

Remark 5.5. The category $\operatorname{Rep}\left(\mathcal{H}_{k}(t)\right)$ has been studied in more detail in the paper $[\mathrm{M}]$.

\subsection{Interpolation of degenerate affine Hecke algebras of type}

B. Let $\mathfrak{h}$ be the reflection representation of the Weyl group $S_{n} \ltimes z_{2}^{n}$ of type $B_{n}$. Let $\Sigma$ be the set of reflections in this group conjugate to a transposition in $S_{n}$, and $\Sigma_{-1}$ the set of reflections conjugate to $(-1,1, \ldots, 1)$. Recall (see e.g. [RS]) that the dAHA $\mathcal{H}_{k_{1}, k_{2}}(n)$ of type $B_{n}$ is the quotient of $\mathrm{C}\left[S_{n} \ltimes \mathrm{Z}_{2}^{n}\right] \ltimes T \mathfrak{h}$ by the relations

$$
\left[y, y^{\prime}\right]=k_{1} \sum_{s, s^{\prime} \in \Sigma}\left(s y, s^{\prime} y^{\prime}\right)_{\mathfrak{h}}\left[s, s^{\prime}\right]+k_{2} \sum_{s \in \Sigma, s^{\prime} \in \Sigma_{-1}}\left(s y, s^{\prime} y^{\prime}\right)_{\mathfrak{h}}\left[s, s^{\prime}\right]
$$

where $(,)_{\mathfrak{h}}$ is the natural inner product on $\mathfrak{h}$.

The object $\mathfrak{h}$ is defined in the category $\operatorname{Rep}\left(S_{t} \ltimes z_{2}^{t}\right)$ for any $t$, and has a natural symmetric pairing $(,)_{\mathfrak{h}}: \mathfrak{h} \otimes \mathfrak{h} \rightarrow \mathbf{1}$. Therefore, similarly to the type $A$ case, we can define the interpolation of the category of modules over $\mathcal{H}_{k_{1}, k_{2}}(n)$. Namely, we make the following

Definition 5.6. Let $t \in \mathrm{C}$. An object of the category $\widehat{\operatorname{Rep}}\left(\mathcal{H}_{k_{1}, k_{2}}(t)\right)$ is an ind-object of Knop's category $\operatorname{Rep}\left(S_{t} \ltimes \mathrm{Z}_{2}^{t}\right)$ equipped with a morphism

$$
y: \mathfrak{h} \otimes M \rightarrow M
$$

such that the morphism

$$
y \circ(1 \otimes y) \circ((1-\sigma) \otimes 1): \mathfrak{h} \otimes \mathfrak{h} \otimes M \rightarrow M,
$$

equals

$$
\left((,)_{\mathfrak{h}} \otimes 1\right) \circ\left(k_{1}\left[\Omega^{13}, \Omega^{23}\right]+k_{2}\left[\Omega^{13}, \Omega_{-1}^{23}\right]\right) .
$$

Morphisms in $\widehat{\operatorname{Rep}}\left(\mathcal{H}_{k_{1}, k_{2}}(t)\right)$ are morphisms in $\operatorname{IndRep}\left(S_{t} \ltimes \mathrm{z}_{2}^{t}\right)$ which respect $y$.

Remark 5.7. Note that similarly to the classical case, $\left(k_{1}, k_{2}\right)$ can be rescaled without changing the category. 
5.3. Interpolation of symplectic reflection algebras for wreath products. Let $\Gamma \subset S L_{2}(\mathrm{C})$ be a finite subgroup. Let $\hbar, k \in \mathrm{C}$, and fix complex numbers $c_{C}$, for nontrivial conjugacy classes $C \subset \Gamma$. Also denote by $T_{C}$ the half-trace of an element $\gamma \in C$ in the tautological representation: $T_{C}=\left.\frac{1}{2} \operatorname{Tr}\right|_{\mathrm{C}^{2}} \gamma$.

Let $V=\left(\mathrm{C}^{2}\right)^{n}$ be the tautological representation of the wreath product $S_{n} \ltimes \Gamma^{n}$. This representation has a natural symplectic pairing $\omega($, ). Let $\Sigma$ be the set of elements conjugate in $S_{n} \ltimes \Gamma^{n}$ to transpositions, and $\Sigma_{C}$ the set of elements of $S_{n} \ltimes \Gamma^{n}$ conjugate to elements of the form $(\gamma, 1,1, \ldots, 1), \gamma \in C$ where $C$ is a nontrivial conjugacy class in $\Gamma$.

Recall ([EG, EGG]) that the symplectic reflection algebra $H_{\hbar, k, c}(\Gamma, n)$ is the quotient of $\mathrm{C}\left[S_{n} \ltimes \Gamma^{n}\right] \ltimes T V$ by the relations

$\left[y, y^{\prime}\right]=\hbar \omega\left(y, y^{\prime}\right)-k \sum_{s \in \Sigma} \omega\left(y,(1-s) y^{\prime}\right) s-\sum_{C} \frac{c_{C}}{1-T_{C}} \sum_{s \in \Sigma_{C}} \omega\left((1-s) y,(1-s) y^{\prime}\right) s$.

The object $V$ is defined in the category $\operatorname{Rep}\left(S_{t} \ltimes \Gamma^{t}\right)$ for any $t$, and has a natural symplectic pairing $\omega: V \otimes V \rightarrow 1$. Therefore, we can define the interpolation of the category of modules over $H_{\hbar, k, c}(\Gamma, n)$. Namely, we make the following

Definition 5.8. Let $t \in \mathrm{C}$. An object of the category $\widehat{\operatorname{Rep}}\left(H_{\hbar, k, c}(\Gamma, t)\right)$ is an ind-object of Knop's category $\operatorname{Rep}\left(S_{t} \ltimes \Gamma^{t}\right)$ equipped with a morphism

$$
y: V \otimes M \rightarrow M,
$$

such that the morphism

$$
y \circ(1 \otimes y) \circ((1-\sigma) \otimes 1): V \otimes V \otimes M \rightarrow M,
$$

equals

$$
(\omega \otimes 1) \circ\left(\hbar-k\left(\Omega^{3}-\Omega^{23}\right)-\sum_{C} \frac{c_{C}}{1-T_{C}}\left(\Omega_{C}^{3}-\Omega_{C}^{13}-\Omega_{C}^{23}+\Omega_{C}^{123}\right)\right) .
$$

Morphisms in $\widehat{\operatorname{Rep}}\left(H_{\hbar, k, c}(\Gamma, n)\right)$ are morphisms in $\operatorname{IndRep}\left(S_{t} \ltimes \Gamma^{t}\right)$ which respect $y$.

Remark 5.9. Note that similarly to the classical case, $(\hbar, k, c)$ can be rescaled without changing the category.

5.4. Interpolation of rational Cherednik algebras of type $A$. It is instructive to consider separately the simplest special case $\Gamma=1$, i.e., that of the rational Cherednik algebra of type $A$. In this case, there is no classes $C, V=\mathfrak{h} \oplus \mathfrak{h}^{*}$ and the definition is simplified 10 Namely, we have

\footnotetext{
${ }^{10}$ To avoid confusion, we do not identify $\mathfrak{h}$ and $\mathfrak{h}^{*}$ here.
} 
Definition 5.10. An object of the category $\widehat{\operatorname{Rep}}\left(H_{\hbar, c}(t)\right)$ is an indobject of Deligne's category $\operatorname{Rep}\left(S_{t}\right)$ equipped with two morphisms

$$
x: \mathfrak{h}^{*} \otimes M \rightarrow M, y: \mathfrak{h} \otimes M \rightarrow M,
$$

satisfying the following conditions:

(i) The morphism

$$
x \circ(1 \otimes x) \circ((1-\sigma) \otimes 1): \mathfrak{h}^{*} \otimes \mathfrak{h}^{*} \otimes M \rightarrow M
$$

is zero;

(ii) The morphism

$$
y \circ(1 \otimes y) \circ((1-\sigma) \otimes 1): \mathfrak{h} \otimes \mathfrak{h} \otimes M \rightarrow M
$$

is zero;

(iii) The morphism

$$
y \circ(1 \otimes x)-x \circ(1 \otimes y) \circ(\sigma \otimes 1): \mathfrak{h} \otimes \mathfrak{h}^{*} \otimes M \rightarrow M,
$$

when regarded as an endomorphism of $\mathfrak{h}^{*} \otimes M$, equals

$$
\hbar-c\left(\Omega^{2}-\Omega^{12}\right) .
$$

Morphisms in this category are morphisms of the underlying ind-objects of $\operatorname{Rep}\left(S_{t}\right)$ respecting $x, y$.

Remark 5.11. The category $\widehat{\operatorname{Rep}}\left(H_{1, c}(t)\right)$ is studied in much more detail in the paper [EA1].

\section{Appendix: Proof of Lemma 2.8}

Let $\mu$ be a Young diagram with $|\mu|=n$, such that $\operatorname{dim} \pi_{\mu} \leq C n^{k}$, and $d$ be the length of its first row. For $1 \leq i \leq d$, let $c_{i}$ be the length of the $d-i+1$-th column of $\mu$. Let $\mu^{\prime}$ be obtained from $\mu$ by deleting the first row. Then by the hooklength formula,

$$
\operatorname{dim} \pi_{\mu}=\operatorname{dim} \pi_{\mu^{\prime}}\left(\begin{array}{l}
n \\
d
\end{array}\right) \prod_{i=1}^{d}\left(1+\frac{c_{i}-1}{i}\right)^{-1} .
$$

Since $\sum c_{i}=n$, by the arithmetic and geometric mean inequality (as in the proof of Claim 1 in Section 3.2.1 of [EFP]), we have

$$
\prod_{i=1}^{d}\left(1+\frac{c_{i}-1}{i}\right) \leq \prod_{i=1}^{d} c_{i} \leq\left(\frac{\sum_{i=1}^{d} c_{i}}{d}\right)^{d}=\left(\frac{n}{d}\right)^{d}
$$

so we get

$$
\operatorname{dim} \pi_{\mu} \geq\left(\begin{array}{l}
n \\
d
\end{array}\right)\left(\frac{n}{d}\right)^{-d}
$$


The same bound holds if $d$ is the length of the first column. Hence, it holds if $d$ is the maximum of the lengths of the first row and the first column of $\mu$, which we assume from now on.

Our job is to show that for large enough $n$, one must have $n-d \leq K$ for some fixed $K$. Then it will follow from the hooklength formula that we actually have $d \geq n-k$ for large $n$.

Since $\operatorname{dim} \pi_{\mu} \leq C n^{k}$, taking logs, we get the inequality

$$
\log \left(\begin{array}{l}
n \\
d
\end{array}\right)-d \log \left(\frac{n}{d}\right) \leq k \log n+\log C .
$$

Let us divide this inequality by $n$, and use Stirling's formula. Setting $x=1-d / n$, after a short calculation we obtain

$$
x \log \frac{1}{x}=O\left(\frac{(k+1 / 2) \log n}{n}\right) .
$$

But we know that $d \geq \sqrt{n}$ (as $\mu$ fits in the $d$ by $d$ square). Hence, $x \leq 1-n^{-1 / 2}$. Formula (3) therefore implies that for large $n, x$ must actually be close to 0 . But then we must have $x=O(1 / n)$, i.e. $n-d=$ $x n$ is bounded, as desired.

\section{REFERENCES}

[CO] J. Comes, V. Ostrik, On blocks of Deligne's category $\operatorname{Rep}\left(S_{t}\right)$, Advances in Math. 226 (2011), 1331-1377, arXiv:0910.5695.

[De1] P. Deligne, Categories Tannakiennes, In The Grothendick Festschrift, Vol. II, Prog. Math. 87 (1990), p.111-195.

[De2] P. Deligne, La Catégorie des Représentations du Groupe Symétrique $S_{t}$, lorsque $t$ n'est pas un Entier Naturel, http://www.math.ias.edu/ phares/deligne/preprints.html

[DM] P. Deligne and J.S. Milne, Tannakian Categories. Hodge cycles, motives, and Shimura varieties. Lecture Notes in Mathematics, 900. Springer, 1982.

[Dr] V. Drinfeld, Degenerate affine Hecke algebras and Yangians, Func. Anal. Appl., 20 (1986), 62-64.

[EFP] D. Ellis, E. Friedgut and H. Pilpel, J. Amer. Math. Soc. 24 (2011), 649-682.

[EA1] Inna Entova-Aizenbud, On representations of rational Cherednik algebras in complex rank, arXiv:1301.0120.

[EA2] Inna Entova-Aizenbud, Schur-Weyl duality for Deligne categories, to appear.

[E] P. Etingof, "Representation theory in complex rank", talk at the Newton Institute of Mathematical Sciences, Cambridge, UK, March 27, 2009, http://www.newton.ac.uk/programmes/ALT/seminars/2009032716301.html

[EG] P. Etingof and V. Ginzburg, Symplectic reflection algebras, Calogero-Moser space, and deformed Harish- Chandra homomorphism, Invent. Math., 147 (2002), pp. 243-348, arXiv:math/0011114.

[EGG] P. Etingof, W.L. Gan and V. Ginzburg, Continuous Hecke algebras, Transform. Groups 10, issues 3-4 (2005), pp. 423-447, arXiv:math/0501192. 
[Kn1] F. Knop, A construction of semisimple tensor categories, C. R. Math. Acad. Sci. Paris 343 (2006) 15-18, arXiv:math/0605126.

[Kn2] F. Knop, Tensor envelopes of regular categories, Adv. Math. 214 (2007) 571617, arXiv:math/0610552.

[M] A. Mathew, Categories parametrized by schemes and representation theory in complex rank, Journal of Algebra Volume 381, 2013, Pages 140163, arXiv:1006.1381

[Mo] M. Mori, On representation categories of wreath products in non-integral rank, arXiv:1105.5091.

[RS] A. Ram and A.V. Shepler, Classification of graded Hecke algebras for complex reflection groups, Comment. Math. Helv. 78 (2) (2003), pp. 308-334.

Department of Mathematics, Massachusetts Institute of Technology, CAMbridge, MA 02139, USA

E-mail address: etingof@math.mit.edu 